\title{
The typology of Mandarin infinitives
}

\section{Cherlon Ussery, Lydia Ding \& Yining Rebecca Liu*}

\begin{abstract}
There has been a long-standing debate in the literature about whether Mandarin has infinitival clauses. Since there is no verbal morphology to distinguish finite and nonfinite clauses, this is an open question. Researchers have used diagnostics such as the availability of an overt embedded subject and the interpretation of aspect markers to argue both for and against the presence of infinitival clauses in Mandarin. Using some of these diagnostics, in addition to the availability of partial control interpretations, Grano (2012/2015) argues that the distinction between types of clauses in Mandarin is not based on finiteness, but rather based on whether there is restructuring: some complement clauses are $v \mathrm{Ps}$, while others are CPs. We provide new data based on the distribution and interpretation of the reflexive ziji, which suggests that there is a finite/nonfinite distinction. We argue for the existence of nonfinite control complements in Mandarin. Further, we evaluate the diagnostics used by previous researchers and illustrate that some of them are not reliable indicators of finiteness or of clause size.
\end{abstract}

Keywords. Mandarin; infinitives; restructuring; partial control; aspect; ziji

1. Introduction. Unlike languages that have even minimal verbal inflection, Mandarin does not morphologically indicate finiteness. Consequently, it is difficult to discern whether Mandarin has infinitival clauses. The sentence in (1) is like its English counterpart in that there is no overt subject in the embedded clause. However, since Mandarin is a pro-drop language, the embedded clause could ostensibly be interpreted as either finite or nonfinite.

(1) Xiaoming xihuan (*ta) chi shousi.

Xiaoming like he eat sushi

'Xiaoming likes to eat sushi./Xiaoming likes that he eats sushi.'

We will show that the embedded clause in (1) is, indeed, nonfinite.

We build on the body of literature that has attempted to answer the question of whether Mandarin distinguishes between finite and nonfinite clauses. Several researchers (e.g., Li 1990; Huang 1987, 1998; Tang 2000; Lin 2011/2012) belong to the Distinction Camp (DC) and argue that Mandarin has both finite and nonfinite clauses. Other researchers (e.g., Hu et al. 2001; Xu 2003) belong to the No-Distinction Camp (NDC), arguing that Mandarin has only one type of clause. We present new data which supports the Distinction Camp.

Of particular interest is Grano's (2012/2015) proposal, which argues that the distinction between different kinds of clauses in Mandarin is actually based on clause size, not on finiteness. Building on the large body of work on restructuring (notably Wurmbrand 2001 and later work), Grano (2012/2015) argues that some complement clauses in Mandarin are full clausal

\footnotetext{
${ }^{*}$ We are thankful to Carleton College for funding this project from Summer 2015 to Winter 2016. Also, thank you to the audience at the $90^{\text {th }}$ Meeting of the Linguistic Society of America. The second and third authors are native speakers of Mandarin and most of the language judgments expressed here are theirs. That said, special thanks to Xiaoyu Gu, Yanshan Guo, Qiyuan Hu, Heqing Huang, Kai Huang, Shatian Wang and Sijia Wang for assistance when we needed additional judgments. All errors are ours. Cherlon Ussery, Carleton College (cussery@carleton.edu), Lydia Ding, Carleton College (dingl@ carleton.edu) \& Yining Rebecca Liu, Carleton College (liur3@carleton.edu).
} 
projections, CPs; other complement clauses are reduced projections, $v$ Ps. On Grano's (2012/2015) proposal, the complement in (1) is a $v \mathrm{P}$, while the complement in (2) is a CP. ${ }^{1}$

(2) Xiaoming $\mathrm{i}_{\mathrm{i}}$ xiwang $\left(\mathrm{ta}_{\mathrm{j}}\right)$ chi shousi.
Xiaoming hope he eat sushi
'Xiaoming hopes to eat sushi./Xiaoming ${ }_{\mathrm{i}}$ hopes that he $\mathrm{j}_{\mathrm{j}}$ (will) eat sushi.'

While a CP- $v$ P distinction may hold in Mandarin, we contribute new data which cannot be solely explained by a clause-size analysis.

Grano (2012/2015) argues that the diagnostics used to distinguish $v \mathrm{P}$ and CP complements in English can be extended to languages which morphologically pattern quite differently from English. ${ }^{2}$ The first diagnostic is whether the embedded subject can be overt. Complement clauses that are CPs allow for an overt embedded subject, while embedded $v$ Ps do not. This is illustrated by the contrast between (1) and (2). When the embedded subject is silent in (2), it has free reference. ${ }^{3}$ (The pronounced subject has disjoint reference from the matrix subject.) Building on Huang (1987), Grano (2012/2015) argues that the subject is pro and not the fixed reference PRO that is present in standard control theory. The second diagnostic is the availability of partial control interpretations. In traditional accounts of infinitives, partial control obtains when PRO refers to a set of individuals which includes the matrix subject. ${ }^{4}$ In sentences such as (3)a, the semantics of 'meet' requires that the embedded subject is a plurality, and in sentences such as (3)b, the adverb 'together' forces a plural subject.

(3) a. The chair of the department ${ }_{i}$ plans $\left[\mathrm{PRO}_{i+j}\right.$ to meet at noon].

b. $\mathrm{John}_{\mathrm{i}}$ hopes $\left[\mathrm{PRO}_{\mathrm{i}+\mathrm{j}}\right.$ to eat lunch together tomorrow].

Grano (2012/2015) proposes that constructions such as (2), which contain embedded CPs, allow partial control interpretations, in which pro constitutes a set. Conversely, constructions such as (1), which contain embedded $v \mathrm{Ps}$, force the embedded subject to be exhaustively referential with the matrix subject. ${ }^{5}$ The third diagnostic is the interpretation of aspect markers. While aspect markers can appear in both kinds of embedded clauses, Grano (2012/2015) argues that aspect markers in embedded $v$ Ps require that the event time of the embedded clause is temporally dependent on the event time of the matrix clause. Aspect markers in embedded CPs, however, do not force a temporal dependence. In (4)b, the perfective marker le and the experiential marker guo entail that the event of eating necessarily precedes the event of telling. By contrast, this entailment does not exist in (4)a; the event of eating does not precede the event of inviting.

a. Zhangsan qing Lisi [chi-le/-guo fan].

embedded $v P$ Zhangsan invite Lisi eat-prf/exp food 'Zhangsan invited Lisi to have a meal.'

b. Zhangsan gaosu Lisi [Wangwu chi-le/-guo fan]. embedded CP Zhangsan tell Lisi Wangwu eat-prf/exp food 'Zhangsan told Lisi that Wangwu had a meal.' (Grano 2015:154)

\footnotetext{
${ }^{1}$ Grano refers to the constructions with $\mathrm{CP}$ complements as biclausal and those with $v \mathrm{P}$ complements as monoclausal. Except in Section 4.2.2, we use the $\mathrm{CP} / v \mathrm{P}$ terminology throughout.

${ }^{2}$ The other language which Grano (2012/2105) discusses in detail is Greek, which is the opposite of Mandarin in that both finite and seeming nonfinite verbs are inflected.

${ }^{3}$ See Huang (1984/1987) for a detailed discussion of the distribution of null pronouns in Mandarin.

${ }^{4}$ See Landau 2000/2013 for detailed discussion of partial control.

${ }^{5}$ There is an exception here, which we discuss in Section 4.1.
} 
Crucially, Grano (2012/2015) argues that the entailment relationships or lack thereof are independent of the semantics of the matrix verbs. In essence, embedded aspect markers inside of $v$ Ps are interpreted with the matrix clause, while aspect markers inside of CPs are interpreted with the embedded clause.

To summarize Grano's (2012/2015) proposal: what has previously been thought to be a distinction in the finiteness of Mandarin embedded clauses is actually a distinction in clause size. $\mathrm{CP}$ complements have the following properties: (1) they have either a null pro subject or an overt subject; (2) they allow for partial control; and (3) they force embedded aspect markers to be associated with the embedded clause. On the other hand, $v \mathrm{P}$ complements have these properties: (1) they do not allow for an overt embedded subject; (2) they do not allow partial control; and (3) they force embedded aspect markers to be associated with the matrix clause. It is important to note that Grano (2012/2015) argues that verbs which select for CPs and those which select for $v$ Ps are mutually exclusive.

Our goal is two-fold. First, we contribute new data based on the distribution and interpretation of ziji, and in doing so, we propose a three-way distinction between types of embedded clauses in Mandarin. Ziji in Mandarin has at least two meanings: it can be a reflexive meaning 'oneself,' or it can be an adverb meaning 'on one's own.' In the sentence in (5), ziji necessarily has the adverbial interpretation, while in (6), ziji has only the reflexive interpretation. Interestingly, ziji is ambiguous in (7), having either the adverbial or the reflexive interpretation.

(5) Type One: only the adverb 'on one's own' interpretation: Nonfinite complement Xiaoming xihuan ziji chi shousi.

Xiaoming like adverb eat sushi

'Xiaoming likes to eat sushi by himself.'

*'Xiaoming likes to eat sushi.'

(6) Type Two: only the reflexive interpretation: Finite complement Xiaoming shuo ziji dai beibao le. Xiaoming say reflexive bring backpack ASP 'Xiaoming says that he brought a backpack.'

*'Xiaoming says that he brought a backpack by himself.'

(7) Type Three: ambiguous, can have either interpretation: Either finite or nonfinite complement Xiaoming wangji ziji dai beibao le.

Xiaoming forget adverb/reflexive bring backpack ASP

Reading 1(adverb): 'Xiaoming forgot to bring the backpack on his own.'

(The backpack was not brought.)

Reading 2 (reflexive): 'Xiaoming forgot that he brought the backpack.'

(The backpack was brought.)

We argue that the best way to account for the data in (5) - (7) is to maintain a finite/nonfinite distinction. Verbs such as xihuan 'like' embed nonfinite complements; verbs such as shuo 'say' embed finite complements; and verbs such as wangji 'forget' embed either finite or nonfinite complements.

Our second goal is to evaluate some of the other diagnostics for clause type discussed above. We show that partial control interpretations can hold in clauses that Grano (2012/2015) argues are $v$ Ps. Additionally, we illustrate the complexity of aspect in Mandarin and show that aspect does not reliably distinguish between types of clauses. 
Before proceeding, a brief discussion about big-picture issues related to control theory is in order. The last decade and a half has seen contentious debate about the nature of infinitival constructions. The Movement Theory of Control (MTC) (notably proposed in Hornstein 1999 and discussed in subsequent work) abolishes PRO (along with the assumption that a DP bears only one theta role); the overt matrix subject is initially merged in the complement clause and moves to the matrix clause. With respect to Mandarin, Grano (2012/2015) also argues that PRO does not exist. He adopts the movement approach for $v \mathrm{P}$ complements and proposes that $\mathrm{CP}$ complements contain the freely referential pro, as noted above. While we, by default, assume PRO in the constructions that we identify as containing control clauses, we do not intend the arguments put forth in this paper as necessarily in opposition to the MTC. Exploring the merits of PRO theory versus the MTC is not our aim; arguing for the existence of nonfinite clauses in Mandarin is our goal. We do, however, illustrate in Section 4.1 that there is an undesirable consequence of Grano's (2012/2015) movement approach with respect to partial control.

The remainder of this paper is organized as follows. Section 2 discusses previous observations about embedded subjects. Section 3 provides a detailed discussion of our analysis, based on ziji and the overall availability and interpretation of embedded subjects. Section 4 evaluates the utility of partial control and embedded aspect as diagnostics. Section 5 discusses open questions with respect to other types of nonfinite constructions in Mandarin. Section 6 concludes.

2. Previous Literature on Embedded Subjects. In this section, we provide a brief overview of the discussion in the literature about what overt embedded subjects reveal about their clauses. As discussed above, for Grano (2012/2015), the allowability of an overt embedded subject is an indicator of clause size. Within the finite/nonfinite debate, the allowability of an overt embedded subject is an indicator of finiteness. While we ultimately side with the Distinction Camp (DC), we suggest that an embedded subject can be pronounced in a nonfinite clause under very particular circumstances.

Because finite $\mathrm{T}$ is believed to assign case to syntactic subjects, a reliable test for finiteness is whether an embedded subject can be overt. In English, there are only two situations where pronouncing the embedded subject of a nonfinite clause is allowed: when case is assigned to the NP by the preposition for, as in (8)a, or when case is assigned by the matrix verb in the ECM construction in (8)b. In other nonfinite contexts, the Case Filter forces the subject to remain silent. DC observes that some Mandarin verbs, such as zhunbei 'prepare' in (9), force the embedded subject to be silent and argue that this is best explained by a nonfinite T's inability to assign case (Huang 1998; Li 1990; Tang 1990). Further, Li (1990) argues that Mandarin has neither a case-marking prepositional complementizer like the English for nor ECM verbs like believe. Therefore, all embedded clauses where the subject is obligatorily silent are nonfinite.

(8) a. I tried*(for) [him to come]

b. I believe [John to be the winner] (Li 1990:22)

(9) wo zhunbei [(*wo) mingtian lai].

I prepare I tomorrow come

'I prepare to come tomorrow.' (Huang 1998:249)

NDC however, points out that the embedded subject NP can be lexicalized if a phonologically heavy adverbial phrase is inserted between the matrix predicate and the 
embedded predicate, such as in (10). ${ }^{6} \mathrm{Hu}$, Pan and $\mathrm{Xu}$ (2001) argue that there is no evidence supporting an explanation in which the verb zhunbei selects for a finite clause in (10) and a nonfinite clause in (9).

(10) wo zhunbei [mingtian xiawu tian hei yihou wo yigeren lai]. I prepare tomorrow afternoon sky dark after I alone come 'I plan to come alone tomorrow afternoon after it gets dark.' (Hu et al. 2001:1131)

Since it seems that the constraint on the occurrence of the NP can be relaxed, Hu et al (2001) argue that there is a lexical-semantic constraint rather than a syntactic one. According to $\mathrm{Hu}$ et al (2001), the interpretation of a pronoun is regulated by an obviation principle: an overt pronoun tends to have disjoint reference from a "close" prominent NP. This explains why the quantifier meigeren 'everyone' cannot be coreferential with tade 'him' in (11), but can be coreferential with tade in (12).

(11) Meigeren $_{\mathrm{i}}$ na-zou le tade $_{*_{\mathrm{i}} / \mathrm{j}}$ shu. everyone take.away his book 'Everyone has taken away his book.'

(12) Meigeren i $_{i}$ cong wo zher na-zou le tade shu. $_{\text {s }}$ everyone from I here take.away his book

'Everyone has taken his own book from me.'

The argument is that the intervening material in both (10) and (12) allows coreference between the (matrix) subject and the pronoun.

Grano (2012/2015) also comments on examples such as (10) and speculates that processing factors influence the acceptability of the embedded subject. Because of the distance between the moved embedded subject and its landing site in the higher clause, the trace is allowed to be pronounced. Grano (2012/2015) argues that the pronounced trace is an instance of copy control, on which we comment further in the discussion of partial control in Section 4.1. However, the position of the adverbial is unclear on Grano's (2012/2015) $v \mathrm{P}$ analysis.

We argue that the complement in (10) is a CP and tentatively posit that the adverbial functions similarly to English for. The overt subject is allowed in (10) because it receives case from the adverbial. In the next section, we illustrate the ways in which overt embedded subjects actually do reveal finiteness.

3. Proposal: Ziji as a "Fake" Reflexive in Control Clauses. In this section, we present new data which support the proposal that Mandarin contains nonfinite clauses. In particular, we discuss how ziji interacts with the semantics of wangji 'forget' and provide compelling evidence that wangji can embed either a finite or a nonfinite complement. Additionally, we discuss the complexity of using ziji to identify object control. Nonetheless, we show that Mandarin likely has object control constructions.

3.1. ZIJI. As discussed in the introduction, ziji has at least two meanings: it can be a reflexive meaning 'oneself,' or it can be an adverb meaning 'on one's own.' When ziji is not in an argument position, it necessarily takes on the adverbial meaning. As we see in (13), Xiaoming overtly occupies the subject position. As such, ziji has the adverbial meaning.

\footnotetext{
${ }^{6}$ The second and third authors have less clear judgments about (10). For us, examples such as these are marginal.
} 
(13) Xiaoming ziji chi shousi.
Xiaoming adverb eat sushi
'Xiaoming eats sushi by himself.'

When ziji appears overtly in the position of the subject of the embedded clause, it illuminates the fact that verbs in Mandarin relevant to the finite/nonfinite debate can pattern in three different ways. The interpretations of the examples in (14), (15) and (18) illustrate our proposed typology of Mandarin verbs.

The first kind of verb forces the adverbial interpretation of ziji. As with the finite clause in (13), in the nonfinite clause in (14) ziji necessarily has the adverb interpretation.

(14) Type 1: only the adverb 'on one's own' interpretation:

Xiaoming xihuan ziji chi shousi.

Xiaoming like adverb eat sushi

'Xiaoming likes to eat sushi by himself.'

Many verbs that are canonically classified as subject control in English allow only the adverb interpretation of ziji. These verbs include: changshi 'try', xiang 'want', dasuan 'intend', jujue 'refuse', zhunbei 'prepare.' With the exception of constructions containing an embedded adverbial, the embedded subject is obligatorily silent. We argue that PRO occupies the subject position in the lower clause. Verbs that we have identified as Type 1 verbs have been used in the previous literature as supporting a finite/nonfinite distinction, as discussed in Section 2.2. Grano (2012/2015) argues that this type of verb takes a $v \mathrm{P}$ complement and that referencing finiteness is unnecessary. Neither the nonfinite analysis nor the $v \mathrm{P}$ analysis allows for an overt embedded subject. On the nonfinite proposal, PRO occupies the embedded subject spot, and on the $v \mathrm{P}$ proposal, the matrix subject starts off in the embedded subject position and moves to the higher clause. Both analyses can explain why ziji in (14) cannot be interpreted as a reflexive and only as an adverb. The embedded subject is filled, either by PRO or by the trace of the matrix subject. Therefore, ziji necessarily occupies a non-argument position. Both kinds of previous analysis also fare well with verbs in our second category.

The second kind of verb allows only the reflexive interpretation, as shown in (15).

(15) Type 2: only the reflexive interpretation:

Xiaoming shuo ziji dai beibao le.

Xiaoming say reflexive bring backpack ASP

'Xiaoming says that he brought a backpack.'

Other verbs that display this pattern include: cai 'guess', renwei 'believe', xiangxin 'believe', xuanbu 'announce', yiwei 'believe erroneously.' As shown in (16), verbs in this category allow for an overt embedded subject. The embedded silent subject in (17) observes the same coreferentiality as the overt embedded pronoun in (16).

(16) Xiaoming $\mathrm{X}_{\mathrm{i}}$ shuo $\mathrm{ta}_{\mathrm{i} / \mathrm{j}}$ jiintian chuqu chifan.

Xiaoming $_{\mathrm{i}}$ say $\mathrm{he}_{\mathrm{i} / \mathrm{j}}$ today out eat

'Xiaoming says that he will eat out today.'

(17) Jiali de mi chi guang le. Xiaoming shuo pro $_{\mathrm{i} / \mathrm{j}}$ jintian chuqu chifan. home DE rice eat empty ASP. Xiaoming ${ }_{\mathrm{i}}$ say $\mathrm{pro}_{\mathrm{i} / \mathrm{j}}$ today out eat

'The rice at home has all been eaten. Xiaoming says that he will eat out today.' 
Previous literature that argues for a finiteness distinction has similarly argued that tell-type verbs, which roughly align with our Type 2 verbs, select for finite clauses (Li 1985/1990; Huang 1998; Tang 1990). On Grano's (2012/2015) analysis, verbs in this category select for a CP complement. We agree with Grano's (2012/2015) argument that here the silent embedded subject is an occurrence of pro-drop; the overtness of the embedded subject does not affect the interpretation. Because the embedded clause allows for an overt embedded subject, we argue that ziji in (15) occupies the subject position, and as such, is interpreted as a true reflexive. While Grano's (2012/2015) proposal could explain the contrast between Type 1 and Type 2 verbs, his analysis incorrectly predicts that Type 3 verbs should not exist.

As shown in (18), ziji is ambiguous and can have either the adverb or reflexive interpretation. One interpretation corresponds to an event in which Xiaoming forgets to bring the backpack on his own, the consequence of which is that the backpack was not brought. The other interpretation corresponds to an event in which Xiaoming forgets that he brought the backpack. In this scenario, he did actually bring the backpack.

(18) Type 3: ambiguous, can have either interpretation:

Xiaoming wangji ziji dai beibao le.

Xiaoming forget adverb/reflexive bring backpack ASP

Reading 1(adverb reading): 'Xiaoming forgot to bring the backpack on his own.'

$=$ The backpack was not brought.

Reading 2 (reflexive reading): 'Xiaoming forgot that he brought the backpack.'

$=$ The backpack was brought.

Other verbs that allow ziji to be ambiguous include: zhidao 'know', jide 'remember', and jueding 'decide.' On the surface, the verbs in the third category behave very similarly to the verbs in the previous category. On Grano's (2012/2015) proposal, these verbs also have CP complements. As illustrated in (19) and (20), these verbs also allow for both an overt or silent embedded subject. Conflating what we have identified as Type 2 and Type 3 verbs, on Grano's (2012/2015) proposal, the overtness of the subject does not affect meaning. However, we observe that for Type 3 verbs, the interpretation does depend on whether the subject is pronounced or silent. In (19), the backpack was not brought when the embedded subject is silent. By contrast, the overt embedded subject in (20) forces the reading in which the backpack was brought.

Xiaoming wangji dai shubao le.
Xiaoming forget bring backpack ASP

'Xiaoming forgot to bring the backpack' (The backpack was not brought.)

Same meaning as Reading 1 (adverb reading) in (18).

(20) Xiaoming wangji ta dai shubao le.

Xiaoming $_{\mathrm{i}}$ forget $\mathrm{he}_{\mathrm{i} / \mathrm{j}}$ bring backpack ASP

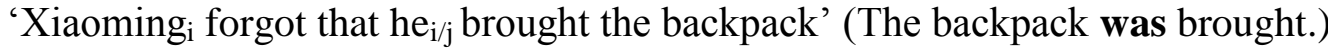

Same meaning as Reading 2 (reflexive reading) in (18).

We argue that the difference in semantics for this group of verbs is best explained with a finite/nonfinite distinction. The interpretations in Mandarin echo the same semantic distinction in the English translations in (19) and (20). As argued in Stowell 1982 (among many others), in English the nonfinite tense refers to an unrealized future. Finite clauses (in English) have an independent tense specification and control infinitives have a tense that is unrealized with respect to the matrix clause. That we see the same contrast in Type 3 verbs suggests that the complement 
clauses are parallel to their English counterparts. In (19) and reading 1 in (18), the embedded clause is nonfinite and has an obligatorily silent subject, which is PRO. This explains the interpretation of ziji as a fake reflexive; it is not in the subject position and acts as an adverb. In (20) and reading 2 in (18), the embedded clause is finite and allows for an overt embedded subject. In this construction ziji is interpreted as a true reflexive co-indexed with Xiaoming.

3.2. OBJECT CONTROL. Our previous discussion has focused on what we argue to be subject control. In this section, we discuss the complexity of identifying object control, which is salient because Grano (2012/2015) uses a variety of ditransitive verbs to illustrate his CP- $v$ P proposal. Based on his other diagnostics, some seeming object control verbs take $\mathrm{CP}$ complements, and others take $v \mathrm{P}$ complements. We show that while the ziji test does not straightforwardly extend to ostensible object control constructions, there is binding evidence which suggests that they are structurally distinct from apparently similar constructions.

In (21), $b i$ 'force' is a canonically object control type verb, while zhidao 'know' in (22) is not. However, on the surface $(21) \mathrm{b}$ and $(22) \mathrm{b}$ seem to have the same structure. In both sentences, ziji has the adverbial 'on one's own' meaning.

(21) a. Gulaoshi bi Xiaoming xue gangqin.

Gu-teacher force Xiaoming learn piano

'Mr. Gu forces Xiaoming to learn piano.'

b. Gulaoshi bi Xiaoming ziji xue gangqin.

Gu-teacher force Xiaoming adverb learn piano

'Mr. Gu forces Xiaoming to learn piano by himself.'

(22) a. Gulaoshi zhidao Xiaoming xue guo gangqin.

Gu-teacher know Xiaoming learn ASP piano

'Mr. Gu knows that Xiaoming has learned piano before.'

b. Gulaoshi zhidao Xiaoming ziji xue guo gangqin.

Gu-teacher know Xiaoming adverb learn ASP piano

'Mr. Gu knows that Xiaoming has learned piano by himself before.' (i.e., Xiaoming is self-taught)'

If the two constructions are actually identical, then one would expect the same binding relationship in (23) and (24). However, this is not the case. In (23)a, the anaphor ta 'he' can be co-indexed with Gulaoshi, Xiaoming, or another person from the discourse. In (24)a, ta can be co-indexed with Gulaoshi or another person, but not with Xiaoming. In (23)b ziji can only be coindexed with Gulaoshi, whereas in (24)b it can be co-indexed with both Gulaoshi and Xiaoming.

(23) a. Gulaoshi $i_{i}$ bi Xiaoming rang $_{\mathbf{j}} \mathbf{t a}_{\mathrm{i} / \mathrm{j} / \mathrm{k}}$ xue gangqin.

Gu-teacher $_{i}$ force Xiaoming let $_{\mathrm{j}} \mathrm{he}_{\mathrm{i} / \mathrm{jk}}$ learn piano

'Mr. Gu forces Xiaoming $_{\mathrm{j}}$ to let $\mathrm{him}_{\mathrm{i} / \mathrm{j} / \mathrm{k}}$ learn piano.'

(co-indexing refers to Mandarin interpretation)

b. Gulaoshi $i_{i}$ bi Xiaoming rang $_{\mathbf{z}} \mathbf{z i j i}_{\mathrm{i} / *_{\mathrm{j}}}$ xue gangqin.

Gu-teacher $_{i}$ force Xiaoming X let $_{j}$ reflexive $_{\mathrm{i} / *_{\mathrm{j}}}$ learn piano

'Mr. $\mathrm{Gu}_{\mathrm{i}}$ forces Xiaoming $\mathrm{j}_{\mathrm{j}}$ to let him $_{\mathrm{i} / *_{\mathrm{j}}}$ learn piano.' (Mandarin interpretation)

(24) a. Gulaoshi $i_{i}$ zhidao Xiaoming rang $\mathbf{t a}_{\mathrm{i} / * \mathrm{j} / \mathrm{k}}$ xue gangqin.

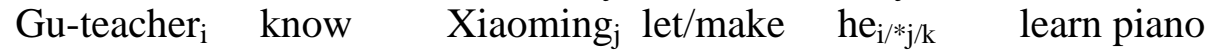

'Mr. $\mathrm{Gu}_{\mathrm{i}}$ knows that Xiaoming let $_{\mathrm{him}}^{\mathrm{i} / *_{\mathrm{j} / \mathrm{k}}}$ learn piano.' (Mandarin interpretation) 
b. Gulaoshi $i_{i}$ zhidao Xiaoming rang $_{\text {ziji }}$ ijj $_{j}$ xue gangqin.

Gu-teacher $_{i}$ know Xiaoming j $_{j}$ let reflexive ${ }_{i j}$ learn piano

'Mr. $\mathrm{Gu}_{\mathrm{i}}$ knows that Xiaoming let $_{\mathrm{j}} \mathrm{him}_{\mathrm{i} / \mathrm{j}}$ learn piano.' (Mandarin interpretation)

This difference in binding suggests different underlying structures. One possible explanation is that (23) contains an object control construction. In (23), Xiaoming acts as the object of the main verb $b i$ 'force' and a silent copy stands as the subject of xue 'learn.' Because this leaves no argument spot available for $z i j i$, it can only behave as an adverb in an adjunct position. By contrast, in (24) Xiaoming is only an argument of xue 'learn.' Since Xiaoming occupies the embedded subject position of the embedded clause, ziji can only be interpreted as an adverb. This explains why, even though on the surface, both constructions show ziji patterning as an adverb, the underlying structure is likely different. We return to Grano's (2012/2015) discussion of object control in our discussion of embedded aspect in section 4.2.

In this section, we have provided evidence which suggests that Mandarin has control structures. We have proposed a novel categorization of verb types in Mandarin based on the interpretation of ziji. Type 1 verbs embed nonfinite complements; Type 2 verbs embed finite complements; and Type 3 verbs embed finite or nonfinite complements. The crucial distinction between Type 2 and Type 3 verbs is that the interpretation depends on the overtness of the embedded subject. In the next section, we turn to previous diagnostics for clause distinctions found in the literature.

4. Other Diagnostics. In addition to the acceptability of an embedded subject, Grano (2012/2015) argues that partial control and the interpretation of aspect markers also distinguish between embedded CP and embedded $v$ Ps. As discussed in Section 2, previous researchers have also based arguments about finiteness on aspect. In this section, we show that neither of these tests reliably distinguishes between different types of embedded clauses.

4.1 PARTIAL CONTROL. Building on previous work on partial control, notably Landau (2001/2013), Grano (2012/2015) argues that the kind of embedded clause which allows partial control is fundamentally different from the kind of clause which disallows partial control. For Landau (2001/2013), this difference is based on the composition of features on the $\mathrm{C}$ and $\mathrm{T}$ heads. For Grano (2012/2015), this difference is based on clause size. The CP complement in (25) allows partial control, while the $v \mathrm{P}$ complement in (26) does not.

(25) Zhangsan xiwang yikuai chi fan.

Zhangsan hope together eat food

'Zhangsan hopes to eat lunch together.' (Grano 2015:144)

(26) *Zhangsan shefa yikuai chi fan.

Zhangsan tried together eat food

'Zhangsan tried to eat together.'

(Grano 2015:144)

We disagree with this judgment; for us (26) is grammatical. Yet, there is a larger issue for the proposal that partial control is an indicator of clause size. Returning to the embedded $v$ Ps which allow an overt subject in the presence of an adverbial, we also find ostensible partial control in constructions such as (27).

(27) Wo dasuan [tian hei yihou women yiqi qu].

I plan sky dark after we together go

'I plan that we go together after it gets dark.' (Grano 2015:148) 
Grano (2015) ${ }^{7}$ argues that (27) exhibits "partial copy control" and that the embedded subject strands part of itself when it moves to the matrix subject position. (Recall that Grano (2012/2015) argues for a movement analysis when the complement is a $v$ P.) Copy control holds when both the controller NP and the controlled NP are pronounced. Polinsky and Potsdam (2006) assume a movement approach to control and suggest that in copy control, the copy is the phonetic realization of the lower portion of the chain. For Grano (2015), it seems that the distinction between canonical partial control and partial copy control is that the former is not allowed with $v \mathrm{P}$ complements but the latter is allowed, provided that there is an overt subject licensed by the adverbial. In essence, the matrix subject can constitute a subset of the embedded subject only if the embedded subject is pronounced. We disagree. Just as we find (26) to be grammatical, we find a partial control interpretation to be available when the embedded subject in (27) is not pronounced. Likewise, Grano's (2015) account predicts (28) to be ungrammatical.

$$
\begin{aligned}
& \text { Wo dasuan mingtian yiqi qu. } \\
& \text { I plan tomorrow together go } \\
& \text { 'I plan to go together tomorrow.' }
\end{aligned}
$$

Given these data, it seems that the availability of partial control interpretations is not a reliable indicator of clause size in Mandarin.

4.2 EMBEDDED ASPECT MARKERS. In this section, we highlight the complexity of aspect in Mandarin. The interpretation of aspect has been used to argue for a finite/nonfinite distinction, as well as for a clause size distinction. Again, we illustrate the unreliability of this diagnostic.

4.2.1 THE FINITENESS DEBATE. Because of the licensing relationship they are argued to have with $\mathrm{T}$, the distribution and interpretation of aspect markers has also been used to identify embedded finite clauses (Li 1985, 1990; Huang 1998). Li (1990) posits that yao and hui are future tense markers in Mandarin. As such, embedded clauses that contain a tense marker are necessarily finite. Similarly, Huang (1998) assumes that modal and aspectual markers belong to AUX and that finite clauses are able to contain an AUX node, while nonfinite clauses cannot. In (29)a, hui 'will' is ungrammatical in the embedded clause, but is grammatical in the matrix clause in (29)b. Huang (1998) takes this contrast to indicate that zhunbei 'prepare' cannot select for a complement clause which contains an embedded AUX.
a. wo zhunbei PRO mingtian (*hui) lai.
I prepare tomorrow will come
'I plan to come tomorrow.'
b. wo hui zhunbei PRO mingtian lai.
I will prepare tomorrow come
'I will plan to come tomorrow.' (Huang 1998:248)

Contra Huang (1998), Hu, Pan, Xu (2001) contend that this ungrammaticality is not due to syntactic constraints, but rather due to semantic incompatibility between the modality of uncertainty encoded in hui and a planned event encoded in zhunbei. In addition, they offer evidence that another modal in Huang's (1998) AUX category, yao, can appear in the embedded clauses for which hui is ungrammatical, as shown in (30). Hu et al (2001) argue that because yao indicates subjective possibility while hui indicates objective possibility, only the former can

\footnotetext{
${ }^{7}$ Grano (2015) addresses partial control in Mandarin more explicitly than Grano (2012). Therefore, we do not cite the 2012 work in some portions of this discussion.
} 
appear with zhunbei 'plan'; planning is subjective, and does not influence the objective probability of an event.

(30) wo zhunbei mingtian yao canjia yige hui.
I plan tomorrow will attend a meeting
'I plan to attend a meeting tomorrow.' (Hu et al 2001:1122, taken from Li 1985)

Hu et al. (2001) show that, as with modals, the placement of aspect markers cannot be used to identify finite clauses. Quan 'persuade' is an object control-type verb that has been argued to select for a nonfinite clause (Huang C. T. J., 1998; Li Y.H.A., 1985, 1990). Yet in (31), le, an aspect marker that Huang takes to be an AUX element, is able to appear in a supposedly nonfinite embedded clause.

(31) wo quan ta chi le zhe wan fan.

I persuade he eat ASP this bowl rice

'I persuade him to finish eating this bowl of rice.'(Hu et al 2001:1122, taken from Li 1985)

We agree with Hu et al's (2001) assertion that these diagnostics only account for a narrow range of data and do not satisfactorily illustrate a finite/nonfinite distinction. Next, we review a proposal by Grano (2012/2015) which relies on both the grammaticality and interpretation of aspectual markers.

4.2.2. THE CLAUSE SIZE PROPOSAL. On Grano's (2012/2015) analysis, embedded aspect markers interpreted with a matrix clause indicate a $v \mathrm{P}$ complement, while embedded aspect markers interpreted with the embedded clause indicate a CP complement. (We should note that in this section, we use the monoclausal/biclausal terminology mentioned in Footnote 1.)

Aspect markers situate the time of an event with respect to the time of the utterance or with respect to the time of an evaluation. For simple sentences such as (32), this means that the matrix event happens before the utterance time.

(32) Lisi he guo jiu.

Lisi drink asp alcohol

'Lisi has drunk the wine before.'

For sentences such as (33)b and (34) (our example), the aspect marker places the time of the embedded event before the matrix time. For sentences such as (33)a, the aspect marker places the event time before the utterance time, just like for simple monoclausal sentences like (32). Therefore (33)a is monoclausal - i.e. the complement is a $v \mathrm{P}$.

(33) a. Zhangsan qing Lisi chi le fan.

Zhangsan invite Lisi eat asp food

Monoclausal/vP complement

' $Z$ invited $L$ to have a meal.'

b. Zhangsan gaosu Lisi Wangwu chi le fan.

Zhangsan tell Lisi Wangwu eat asp food

'Zhangsan told Lisi that Wangwu had a meal.' (Grano 2015:153)

(34) Zhangsan jide Wangwu chi le fan.

Zhangsan remember Wangwu eat asp food

'Zhangsan remembers that Wangwu had a meal.' 
Grano (2012/2015) argues that embedded aspect markers that are associated with the matrix verb indicate what is actually a monoclausal structure. In other words, the semantics of the embedded aspect marker can be used to distinguish between monoclausal and biclausal structures. While this test holds for the object control-type examples that Grano (2012/2015) uses, it does not apply as well to subject control-type verbs. We can think of only one such verb, kaishi 'begin,' that allows an embedded aspect marker. Gan 'dare', for instance, does not allow an embedded aspect marker at all, as shown by the contrast between (35)a and (35)b.

(35) a. Ta kaishi huifu le jiankang.
3sg begin recover asp health
'He began to recover his health.'
b. *Ta gan huifu le jiankang.
3sg dare recover asp health
* 'He dared to recover his health.'

Since this test is limited to object control, it is not a reliable diagnostic of clause size.

Further, in Grano's (2012/2015) system in which a matrix verb can take either only a monoclausal or a biclausal complement, there is not an explanation for the ambiguity in (36)a. The semantics of wangji 'forget' are instructive again. One interpretation is that she forgot that she brought the backpack, where the event time precedes matrix time. In this reading, -le is associated with bringing rather than forgetting. The second interpretation is that she forgot to bring the backpack. Here, the event time of forgetting occurs before utterance time, and -le is associated with forgetting rather than bringing. This second interpretation has the same meaning as (36)b, in which the -le modifies the matrix verb.

(36) a.Ta wangji dai le shubao.

3sg forget bring asp backpack

'She forgot that she brought the backpack' / 'She forgot to bring the backpack.'

b. Ta wangji le dai shubao.

3 sg forget asp bring backpack

'She forgot to bring the backpack.'

If aspect markers can indicate clause size, then the ambiguity in (36)a would contradict Grano's (2012/2015) proposal that a verb cannot select for both biclausal and monoclausal structures.

Mandarin also allows -le to appear at the ends of clauses. Grano (2012/2015) argues that biclausal structures with clause-final -le have ambiguous temporal interpretations, as shown in (37). In the monoclausal structure in (38), on the other hand, clause-final -le is necessarily associated with the matrix clause.

$$
\begin{array}{lllll}
\text { Zhangsan zhidao wo yao qu le. } & \text { Biclausal/CP complement } \\
\text { Zhangsan know } & 1 \mathrm{sg} \text { mod go asp } &
\end{array}
$$

Reading 1: le associated with matrix clause:

'It has now become the case that Zhangsan knows that I'm going to go.'

Reading 2: le associated with embedded clause:

'Zhangsan knows that it has now become the case that I'm going to go.' (Grano 2015:166) 
Zhangsan shefa kai men le.

Zhangsan try open door asp

Monoclausal/vP complement

Reading 1: -le associated with matrix clause:

'It has now become the case the Zhangsan tried to open the door.'

* Reading 2: -le associated with embedded clause:

* 'Zhangsan tried to make it now be the case that he opened the door.' (Grano 2015:168)

Grano (2012/2015) also proposes that clause-final -le blocks restructuring for $\nu \mathrm{P}$-selecting verbs when -le is attached to the matrix verb. In (39), for instance, he argues that the -le attached to qing 'invite' forces that sentence, which would normally be monoclausal, to be biclausal. As such, we expect an attachment ambiguity if clause-final -le is added to a sentence like (39); (39) should pattern like (37). However, (40) demonstrates that there is no such ambiguity; the matrix event of the invitation is necessarily the event that -le places before the utterance time. This suggests either that -le cannot block restructuring, or that the attachment ambiguity of clausefinal -le is an insufficient test for biclausality.

Zhangsan qing le Lisi chi fan.
Zhangsan invite asp Lisi eat food
'Zhangsan invited Lisi to have a meal.

'Zhangsan invited Lisi to have a meal.'

Zhangsan qing le Lisi chi fan le.

Zhangsan invite asp Lisi eat food asp

Reading 1: -le associated with matrix clause:

'It has now become the case that Zhangsan invited Lisi to have a meal.'

*Reading 2: -le associated with embedded clause:

* 'Zhangsan invited Lisi so that it has now become the case that she had a meal.'

In this section, we have demonstrated that neither the availability of partial control nor the interpretation of embedded aspect markers is a reliable indicator of clause size. In the next section, we explore whether Mandarin has raising and/or ECM structures.

5. Open Question: Does Mandarin have Raising or ECM? One test which distinguishes raising from control in English is the distribution of WH words. In English control constructions, a WH word can appear in either the matrix or the embedded clause and the interpretation corresponds to the position of the WH. In (41)a, how scopes over eat and in (41)b, how scopes over know. As shown in (42), Mandarin displays a similar pattern.

(41) a. Yining knows how to eat chocolate.

b. How does Yining know to eat chocolate?

(42) a. Zhangsan zenme dasuan chi qiaokeli

Zhangsan how plan eat chocolate

'How does Zhangsan plan to eat the chocolate?'

b. Zhangsan dasuan zenme chi qiaokeli

Zhangsan plan how eat chocolate

'Zhangsan plans to eat the chocolate how?'

English raising constructions, however, only allow for the WH to appear in the matrix clause, and there is no clear scope distinction. In (43)b, how could refer to either seem or get fat. 
(43) a. *Alex seems how to have gotten fat.

b. How does Alex seem to have gotten fat?

The absence of the embedded WH in (43)a is readily explained by the proposal that raising (and ECM) constructions in English do not contain the functional structure to host the WH, notably proposed in Stowell 1982. In contemporary theories, control clauses are CPs while raising and ECM clauses are TPs.

In Mandarin, however, ostensible raising constructions pattern differently than in English. As with Mandarin control constructions, the WH can appear before either the matrix or the embedded verb and the interpretation corresponds with the position of the WH, just as it does in Mandarin (and English) control constructions. A possible response to the question in (44)a would explain why the speaker thinks that Xixi seems fat. For example, his/her clothes don't fit. Zenme can also take scope over only the lower clause. In (44)b, zenme takes scope over only zhangpang 'get fat.' A possible response would be that Xixi didn't exercise and ate a lot of junk food.

(44) a. Xixi zenme kanqilai zhangpang le

Xixi how seem grow fat ASP

'How does Xixi seem to have gotten fat?'

b. Xixi kanqilai zenme zhangpang le

Xixi seem how grow fat ASP

'How does Xixi seem to have gotten fat?'

Assuming an LF movement approach to WH in situ languages such as Mandarin (Huang 1982), the fact that the WH can scope over just the lower clause suggests that if Mandarin has raising, those clauses are structurally larger than their English counterparts.

$\mathrm{Li}$ (1990) argues that there are no ECM verbs in Mandarin like the English verb 'believe.' We agree with this analysis. Since Mandarin has no overt case marking, we can see in (45) that there is no distinction between the embedded clauses of verbs that are cross-linguistically ECM (such as xiangxin/renwei 'believe', faxian 'find', qiwang 'expect' and xiang 'want') and verbs that are not (such as shuo 'say'). The ziji test also fails to show a difference in behavior. In both believe-type verbs and the say-type verbs, ziji in the embedded subject position only allows for the reflexive interpretation, suggesting that the embedded clauses are finite and that the verbs belong to Type 2 as described in Section 3.1.
a. Xixi xiangxin/renwei [ta dai beibao le]. Xixi believe he bring backpack ASP
'Xixi believes that he brought a backpack.'
b. Xixi shuo [ta dai beibao le]. Xixi say he bring backpack ASP
'Xixi says that he brought a backpack.'

Given the lack of evidence suggesting that the believe-type verbs and the say-type verbs behave differently, there seems to be insufficient evidence supporting an argument for ECM-type verbs in Mandarin.

6. Conclusion. This paper contributes to the literature which argues for a finite/nonfinite distinction in Mandarin. We show that Mandarin verbs are divided into three categories: Type 1 embeds nonfinite complements; Type 2 embeds finite complements; and Type 3 embeds either a finite or a nonfinite complement. Crucially, we have illustrated that the overtness of the 
embedded subject determines the interpretation of constructions with Type 3 verbs. Additionally, we provide evidence which suggests the existence of object control. Further, we show that neither partial control nor embedded aspect can be used as a diagnostic of finiteness or clause size. Finally, we illustrate that Mandarin does not have ECM and that raising to subject constructions have functionally larger embedded clauses than their English counterparts do.

\section{References}

Grano, Thomas. 2012. Control and restructuring at the syntax-semantics interface. Chicago, IL: University of Chicago dissertation.

Grano, Thomas. 2015. Control and restructuring. Oxford: Oxford University Press.

Hornstein, Norbert. 1999. Movement and control. Linguistic Inquiry 30(1). 69-96.

$\mathrm{Hu}$, Jianhua, Haihua Pan \& Liejiong Xu. 2001. Is there a finite vs. nonfinite distinction in Chinese?. Linguistics 39(6). 1117-1148.

Huang, C.-T. James. 1982. Move WH in a language without Wh-movement. The Linguistic Review 1:369-416

Huang, C.-T. James. 1984. On the distribution and reference of empty pronouns. Linguistic Inquiry 15(4):531-574.

Huang, C.-T. James. 1987. Remarks on empty categories in Chinese. Linguistic Inquiry 18(2):321-337.

Huang, C.-T. James. 1998. Logical relations in Chinese and the theory of grammar. New York \& London: Taylor \& Francis.

Huang, Yan. 1994. The syntax and pragmatics of anaphora: A study with special reference to Chinese. Cambridge: Cambridge University Press.

Landau, Idan, 2000. Elements of control: structure and meaning in infinitival constructions. Dordrecht: Kluwer Academic Publishers.

Landau, Idan. 2013. Control in generative grammar: a research companion. Cambridge: Cambridge University Press.

Li, Y.-H. Audrey. 1985. Abstract case in Chinese. Los Angeles CA: University of Southern California dissertation.

Li, Y.-H. Audrey. 1990. Order and constituency in Mandarin Chinese. Dordrecht: Kluwer.

Lin, Tzong Hong Jonah. 2011. Finiteness of clauses and raising of arguments in Mandarin Chinese. Syntax 14(1). 48-73.

Lin, Tzong Hong Jonah. 2012. Multiple-modal constructions in Mandarin Chinese and their finiteness properties. Journal of Linguistics 48(01). 151-186.

Polinsky, Maria \& Eric Potsdam. 2006. Expanding the scope of control and raising. Syntax 9(2):171-192.

Stowell, Tim. 1982. The tense of infinitives. Linguistic Inquiry 13(3). 561-570.

Tang, Chih-Chen Jane. 1990. Chinese phrase structure and the extended $x^{\prime}$-theory. Ithaca, NY: Cornell University dissertation.

Tang, Ting-chi. 2000. Finite and nonfinite clauses in Chinese. Language and Linguistics 1(1). 191-214.

$\mathrm{Xu}$, Liejong. 2003 Choice between the overt and the covert. Transactions of the Philological Society 101(1). 81-107.

Wurmbrand, Susi. 2001. Infinitives: restructuring and clause structure. Berlin: Mouton de Gruyter. 